\title{
Search for heavy resonances in vector boson fusion
}

\author{
Guangyi Zhang ${ }^{1,2, a}$ On behalf of the ATLAS Collaboration \\ ${ }^{1}$ University of Science and Technology of China, Hefei, China \\ ${ }^{2}$ Institute of Physics, Academia Sinica, Taiwan
}

\begin{abstract}
If the Higgs boson discovered at the Large Hadron Collider (LHC) is not exactly the one in the Standard Model, an alternative mechanism is needed to restore unitarity in the scattering amplitude of longitudinal gauge bosons, and new resonances may appear. This paper presents a search for new heavy neutral resonances $(R)$ produced through vector boson fusion process $q q \rightarrow R q q \rightarrow \ell^{+} v \ell^{-} \bar{v} q q(\ell=e, \mu)$ using $3.2 \mathrm{fb}^{-1}$ of data at $\sqrt{s}=13 \mathrm{TeV}$ recorded by the ATLAS detector at the LHC. No excess above the Standard Model background expectation is observed. Limits are set on the production of five types of neutral resonances with different spin and isospin quantum numbers using a $K$-matrix unitarization of the vector boson scattering process.
\end{abstract}

\section{Introduction}

The process of vector boson scattering can be sensitive to phenomena beyond the Standard Model (SM), in particular the presence of diboson resonances and anomalous quartic couplings. Resonances of different spin and isospin quantum numbers are generically predicted in composite Higgs models [1, 2], triplet Higgs models [3-5], and extra dimension models [6, 7]. Cross sections and branching ratios of the $125 \mathrm{GeV}$ scalar boson discovered at the Large Hadron Collider (LHC) are presently compatible with those predicted for the SM Higgs boson. However, if the values of the Higgs coupling to the vector bosons do not take the exact values predicted by the SM, an alternative mechanism is needed to restore unitarity in the scattering amplitude of longitudinal gauge bosons, and new resonances may appear [8-11]. If these resonances have weak or no coupling to fermions, vector boson scattering processes could provide the best sensitivity to these resonance searches at the LHC.

In this paper, a benchmark model is used which combines new resonances of different spin and isospin quantum numbers with an effective chiral Lagrangian (EChL) [12-16]. These resonances are assumed to only couple to longitudinal vector bosons and do not couple to fermions, photons and transverse components of vector bosons. The coupling $(g)$ of the new resonance to the longitudinal vector bosons is set to $g=2.5$ [15]. The resonance decay widths shown in Table 1 are calculated according to Ref. [14]. These resonances contribute additional terms to the scattering amplitudes and are associated with anomalous quartic gauge couplings implying a violation of unitarity. These effects are evaluated in the EChL model and a unitarization procedure is required, as in Refs. [10, 13-16]. Here, a $K$-matrix unitarization procedure [13] is used. The EChL gathers three gauge bosons $\omega^{a}$

ae-mail: g.zhang@cern.ch 
$(a=1,2,3)$ in a matrix which transforms in an $S U(2)_{L} \times U(1)_{Y}$ invariant way and the Higgs boson is included in a nonlinear representation.

A search is performed for the first time for neutral resonances above the Higgs boson mass produced by the $q q \rightarrow R q q \rightarrow \ell^{+} v \ell^{-} \bar{v} q q(\ell=e, \mu)$ fusion process, taking into account interference with the SM production process of the same final state. A representative Feynman diagram of the production and decay of the resonance is shown in Figure 1. Only leptonic channels are considered in view of the relatively small SM backgrounds expected. Three channels are labeled based on the lepton flavors: $e e, e \mu$, and $\mu \mu$. The analysis is based on an integrated luminosity of $3.2 \mathrm{fb}^{-1}$ of proton-proton collision data taken at $\sqrt{s}=13 \mathrm{TeV}$ with the ATLAS detector at the LHC in 2015. The method and results of this analysis are referenced from this ATLAS conference note [17].

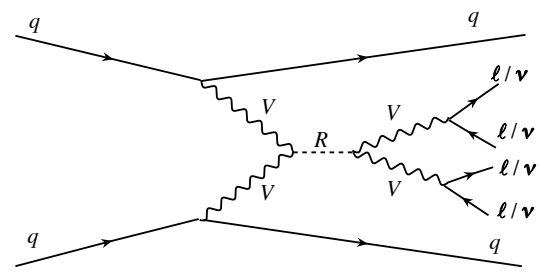

Figure 1. Representative Feynman diagram [17] for the production and decay of a heavy neutral resonance $(R)$ by vector boson fusion at the LHC. The symbol $q$ represents all possible quarks or anti-quarks, and $V$ represents $W$ or $Z$ boson.

Table 1. Spin, isospin and corresponding electric charge for five types of resonances [17]. Only neutral resonances are considered. The last column gives the relative width $\Gamma / \Gamma_{0}$ where $\Gamma_{0}=g^{2} m^{3} / 64 \pi v^{2}$ with $g$ as the coupling of the new resonance to the longitudinal vector bosons [14], $m$ as the mass of the resonance, and $v$ as the electroweak scale of $246 \mathrm{GeV}$.

\begin{tabular}{ccccc}
\hline Type & Spin $J$ & Isospin $I$ & Electric Charge & $\Gamma / \Gamma_{0}$ \\
\hline$\sigma$ & 0 & 0 & 0 & 6 \\
$\phi$ & 0 & 2 & $--,-, 0,+,++$ & 1 \\
$\rho$ & 1 & 1 & $-, 0,+$ & $\frac{4}{3}\left(\frac{v^{2}}{m^{2}}\right)$ \\
$f$ & 2 & 0 & 0 & $\frac{1}{5}$ \\
$t$ & 2 & 2 & $--,-, 0,+,++$ & $\frac{1}{30}$ \\
\hline
\end{tabular}

\section{Data and Simulated Events}

The ATLAS detector [18] is a multi-purpose particle physics apparatus with a forward-backward symmetric cylindrical geometry and almost $4 \pi$ coverage in solid angle. The data were recorded with the ATLAS detector during the 2015 run and correspond to a total integrated luminosity of $3.2 \mathrm{fb}^{-1}$ at a center-of-mass energy of $13 \mathrm{TeV}$. Events are selected during the data-taking with a set of single electron or muon triggers with $p_{T}$ thresholds of $24 \mathrm{GeV}$ for the electron and $20 \mathrm{GeV}$ for the muon.

Simulated Monte Carlo (MC) signal samples, $q q \rightarrow \ell^{+} v \ell^{-} \bar{v} q q(\ell=e, \mu)$, including a new resonance, the contribution from the SM electroweak processes that produce the same final state, and 
the interference between the SM and the new resonance, are generated with the Whizard generator v2.1.1 $[19,20]$. Five types of new neutral resonance, as shown in Table 1, are produced: scalar isoscalar $(\sigma)$, scalar isotensor $(\phi)$, vector isovector $(\rho)$, tensor isoscalar $(f)$ and tensor isotensor $(t)$. Resonance masses ranging from $200 \mathrm{GeV}$ to $500 \mathrm{GeV}$ in steps of $100 \mathrm{GeV}$ are produced. The coupling of the resonance to longitudinal vector bosons is set to $g=2.5$. The vector bosons from the resonance decay are required to be on-shell, thus excluding the $s$-channel Higgs boson exchange contribution, where $H \rightarrow W W^{*}$ and $H \rightarrow Z Z^{*}$.

The signal cross section is defined here formally as the difference in the cross section obtained for the full MC samples of $q q \rightarrow R q q \rightarrow \ell^{+} v \ell^{-} \bar{v} q q$ (which includes the new resonance, the SM background processes producing the same final state, and the interference between the SM and the new resonance) and that of the pure electroweak sample $q q \rightarrow \ell^{+} v \ell^{-} \bar{v} q q$, also generated with Whizard. For $\sigma$ resonances above $500 \mathrm{GeV}$, the ratio of the decay width to the resonance pole mass $(\Gamma / \mathrm{m})$ reaches around $50 \%$ for a chosen coupling of $g=2.5$ [15]. For higher masses the definition of a resonance is difficult due to the large decay width, thus only resonances with mass below $500 \mathrm{GeV}$ are considered.

SM background processes include those that lead to a final state with two opposite-sign prompt leptons and at least two jets, such as $t \bar{t}, Z+$ jets, diboson, and Higgs production. Their contributions are estimated using simulated events. Both $t \bar{t}$ and single top quark production samples are generated with Powheg-Box v2 [21], and the $t \bar{t}+W / Z$ samples are generated using Madgraph v2.2.2 [22]. The $Z\left(\rightarrow \ell^{+} \ell^{-}\right)+$jets QCD production is also generated with Madgraph v2.2.2, and the $Z\left(\rightarrow \ell^{+} \ell^{-}\right)+$jets production by electroweak diagrams $\left(O\left(\alpha^{4}\right)\right)$ is generated with Sherpa2.1.1 [23]. The $W^{+}\left(\rightarrow \ell^{+} v\right) W^{-}\left(\rightarrow \ell^{-} \bar{v}\right) j j$ and other diboson processes in association with jets, $W Z j j, Z Z j j$ and $Z \gamma j j$, produced by QCD diagrams $\left(O\left(\alpha_{s}^{2} \alpha^{2}\right)\right)$ are modelled with Sherpa2.1.1, and the production of the $W W j j$ process by electroweak diagrams $\left(O\left(\alpha^{4}\right)\right)$, including vector boson scattering, is generated with Whizard v2.1.1. Simulation of Higgs production by gluon fusion $\left(g g \rightarrow H\left(\rightarrow W W^{*} \rightarrow \ell v \ell v\right)\right.$ ), denoted as $g g H$, and by vector boson fusion $\left(q q^{\prime} \rightarrow q q^{\prime} H\left(\rightarrow W W^{*} \rightarrow \ell v \ell v\right)\right)$ are generated with Powheg-Box v2.

For the dominant backgrounds, the cross sections of $Z+$ jets processes are normalized to the Nextto-Next-to-Leading Order (NNLO) calculation [24-26], and the cross section of $t \bar{t}$ is normalized to the NNLO calculation including soft-gluon resummation to Next-to-Next-to-Leading-Logarithm order (NNLL) using a $k$-factor [27]. Other generated samples are normalized to the most accurate available cross-section calculations. All generated samples are processed through the Geant4-based ATLAS detector simulation $[28,29]$ and reconstructed with the standard ATLAS reconstruction software used for collision data.

\section{Object and Event Selection}

Candidate events are required to have at least one primary vertex reconstructed with at least two associated tracks with $p_{T}>400 \mathrm{MeV}$ [30]. If an event has more than one such vertex, the vertex with the largest $\Sigma p_{T}^{2}$ of associated tracks is denoted as the primary vertex.

Electrons are required to originate from the primary vertex, to satisfy the "tight" ("medium" used if $E_{T}>300 \mathrm{GeV}$ ) likelihood identification criteria described in [31] and the "tight" isolation requirements [31], and to have transverse momentum $p_{T}>25 \mathrm{GeV}$ and pseudorapidity $|\eta|<2.47$, with a veto in the range $1.37<|\eta|<1.52$ which corresponds to the crack region between the barrel and endcap LAr calorimeters. Candidate muons are required to originate from the primary vertex, satisfy the "medium" identification criteria [32] and "tight" isolation requirements [32], and to have transverse momentum $p_{T}>25 \mathrm{GeV}$ and $|\eta|<2.5$.

Jets are reconstructed using the anti- $k_{t}$ algorithm [33] with a radius parameter of $R=0.4$, using calorimeter clusters as input. Jet energies are calibrated [34] for the impacts of the non-compensating 
nature of the calorimeter, out-of-cluster energy losses and the effect from pile-up. Jets are required to have $p_{T}>30 \mathrm{GeV}$ and $|\eta|<4.5$. A jet vertex tagger (JVT) [35] is a likelihood variable employed to tag and suppress pile-up jets, having a large fraction of tracks not associated with the primary vertex. The ATLAS jet flavor tagging algorithm, MV2c20 [36], with an efficiency of $85 \%$ for tagging $b$-jets is used. The vector boson scattering signal process contains two forward tag jets, which could be contaminated by pile-up jets. To suppress pile-up effect, a higher $p_{T}$ threshold of $50 \mathrm{GeV}$ is set for jets which fall in $|\eta|>2.5$. The missing transverse energy $E_{T}^{\text {miss }}$ [37] is calculated as the negative vector sum of the calibrated transverse momenta from reconstructed objects such as electrons, muons, and jets. Tracks not associated to these hard objects are also taken into account as the track soft term.

Candidate events have a distinct experimental signature of exactly two high $p_{T}$ leptons (electrons or muons), missing transverse energy $\left(E_{T}^{m i s s}\right)$, and at least two forward jets. The two high $p_{T}$ leptons are required to be opposite-sign, to pass the above selection criteria, and to have $m_{\ell \ell}>40 \mathrm{GeV}$. At least one of the two leptons is required to be geometrically matched to a lepton reconstructed by the trigger algorithm. The dilepton invariant mass must not be within $25 \mathrm{GeV}$ of the $Z$ boson mass to reduce $Z+$ jets background for both $e e$ and $\mu \mu$ channels. Events are also required to have $E_{T}^{m i s s}>35 \mathrm{GeV}$. In order to reduce the top quark pair and single top quark background, events must not contain any $b$-jet.

In addition, the two jets must have an invariant mass $m_{j j}$ that is greater than $500 \mathrm{GeV}$ and large separation in pseudorapidities $\left(\left|\Delta \eta_{j j}\right|>2.4\right)$. They are also required to be present in the opposite sides of the detector $\left(\eta_{j_{1}} \times \eta_{j_{2}}<0\right)$. To reduce the background from strong production of double vector boson processes, the lepton centrality is required to have $\zeta>-0.5$. The centrality variable [15] is defined as $\zeta=\min \left(\eta_{1}^{j e t}-\eta_{1}^{\ell}, \eta_{2}^{j e t}-\eta_{2}^{\ell}\right)$, where the leptons and jets are ordered such that $\eta_{1}^{\ell} \geq \eta_{2}^{\ell}$ and $\eta_{1}^{j e t} \geq \eta_{2}^{j e t}$. In order to reject the $Z / \gamma^{*} \rightarrow \ell \ell$ background with misreconstructed $E_{T}^{\text {miss }}$ the variable $f_{\text {recoil }}$ [38] is introduced. The two leptons in the $Z \rightarrow \ell \ell$ events need to be balanced by a hadronic recoil system. The definition of $f_{\text {recoil }}$ is $\left|\sum_{\text {soft jets }} \mathrm{JVT}_{j} \cdot \vec{p}_{\mathrm{T}}^{j}\right| / p_{\mathrm{T}}^{\ell \ell}$, where $p_{\mathrm{T}}^{\ell \ell}$ is the transverse momentum of the dilepton system, $\vec{p}_{\mathrm{T}}^{j}$ is the transverse momentum of the $j$-th soft recoil jet. The soft recoil system is reconstructed as the vector sum $\vec{p}_{\mathrm{T}}$ of soft jets with $p_{T}>10 \mathrm{GeV}$ and $|\eta|<4.5$ in the transverse quadrant opposite the dilepton system. This variable measures the strength of the recoil system relative to the dilepton system, with the $p_{\mathrm{T}}$ of the soft jets weighted by JVT to reduce the effects of soft jets from pile-up. Candidate events are required to have $f_{\text {recoil }}<2.0$.

These selection criteria are applied for five resonance types and masses considered. The signal acceptance times efficiency is in the range of $5 \%-22 \%$ depending on the mass and type of resonance and the decay channel.

\section{Background Estimation and Validation}

The SM backgrounds come from two sources: (1) SM processes with two prompt opposite-sign leptons in the final states and (2) SM processes accompanied by jets that are misidentified as isolated leptons.

Contributions from background processes with two prompt opposite-sign leptons in the final state are estimated using MC-simulated events. These processes include top pair production, $W t$ production, $t \bar{t} V, Z+$ jets, diboson, and Higgs production via gluon fusion and VBF. Processes with a single prompt lepton produced in association with hadronic jets enter the signal region when one jet is misidentified as an isolated lepton. This fake-lepton background mainly originates from $W+$ jets, multijet (including semileptonic decays of $b$-quark pairs) and semileptonic decay of the $t \bar{t}$ processes. Since the probability for a jet to be misidentified as an isolated lepton may not be accurately mod- 
elled in the MC simulation, a data-driven technique, called the "matrix method" [39], is employed to estimate this contribution.

The probability for a jet to be misidentified as an isolated muon is found to be small given the muon selection criteria used, thus only jets misidentified as electrons are considered. The matrix method uses the probabilities for a looser quality electron to pass the default electron selection for both prompt and non-prompt leptons. These two probabilities are called efficiency and fake probability, and are measured in data using $Z \rightarrow e e$ events and dijet events. The electron average efficiency is found to be around $84 \%$ and the average fake probability is found to be $13 \%$. The matrix method is applied on each event and the resulting weights are then summed over all events to obtain the fake-lepton background.

With all selection criteria applied, the $Z+$ jets process is the dominant background source in the $e e$ and $\mu \mu$ channels while $t \bar{t}$ is the main background source in the $e \mu$ channel. The $Z$ boson $p_{T}$ is found to be not modelled well with the event generator used, so a reweighting function is derived to correct the $\mathrm{MC}$ prediction to match with the observed $Z$ boson $p_{T}$ distribution in data. This reweighting function is used for the $Z+$ jets prediction in both validation and signal regions.

Three validation regions (VRs), referred to as the " $Z+$ jets VR", " $t \bar{t}$ VR", and "low $-m_{j j}$ VR", are used to validate background predictions. Three VRs select phase spaces similar to the one corresponding to the signal region, with only one or two selection criteria loosened or inverted. The signal contribution to these VRs is negligible. The $Z+$ jets VR is used to test the $Z+$ jets background prediction and the dilepton mass is required to be within $25 \mathrm{GeV}$ of the $Z$ boson pole mass. The $t \bar{t} \mathrm{VR}$ is used to test the $t \bar{t}$ background estimation, and is defined by inverting the $b$-jet veto criteria to require the presence of at least one $b$-tagged jet in the event. The $m_{j j}$ selection criteria is dropped in both VRs to obtain more statistics. The low $m_{j j}$ VR is used to check the background modelling in a region with similar background composition to the signal region, and is defined by requiring $m_{j j}<500 \mathrm{GeV}$.

Good agreement between data and predictions is seen in all validation regions. Table 2 shows the number of data events compared to the expectations from signal and various background sources for the "low- $m_{j j}$ VR". Backgrounds from similar sources are grouped together. The term " $Z+$ jets" includes both electroweak and strong production of $Z+$ jets processes, " $t \vec{t}$ ' and " $W t$ " represent the top pair and single top production, respectively. The term "diboson_QCD" includes strong production of $\ell \ell v v(W W / Z Z), W(\rightarrow q q) Z(\rightarrow \ell \ell)$ and $Z(\rightarrow q q) Z(\rightarrow \ell \ell)$, and "diboson_EW" includes electroweak production of $W W j j$ and $Z Z j j$. The term " $Z \gamma$ " represents the production of $Z \gamma j j$, while " $t \bar{t} V$ " includes both $t \bar{t} W$ and $t t Z$ contribution. The term "Higgs" includes both gluon-fusion (ggH) and VBF processes.

Experimental systematic uncertainties on the signal and background yields estimated from MC simulation are derived from uncertainties on object reconstruction and identification efficiency correction factors, energy and momentum scaling and smearing parameters, the $E_{T}^{\text {miss }}$ modelling, and the $b$-tagging efficiency/mistag rate, luminosity and trigger efficiency. The dominant uncertainties come from uncertainties on jet energy scale (JES) and jet energy resolution (JER). Uncertainties on the $Z+$ jets background due to the $Z$ boson $p_{T}$ reweighting and the uncertainty on the fake-lepton background estimate using the matrix method are also taken into account. The overall experimental systematic uncertainty on the background yields is found to be $35 \%, 31 \%$, and $20 \%$ in the $e e, \mu \mu$, and $e \mu$ channels, respectively. Theoretical uncertainties on the production cross sections of various physics processes with contributions estimated from $\mathrm{MC}$ simulation are included, ranging from $5 \%$ to $20 \%$ according to the process. Additional shape systematic uncertainties are considered for the two dominant background sources $(Z+$ jets, $t \bar{t})$ by comparing results from different generators. Depending on the resonance type and the pole mass, the overall systematic uncertainty on the signal yield varies 
Table 2. Background estimation and data yields [17] in the low- $m_{j j} \mathrm{VR}$ for the $e e, e \mu$ and $\mu \mu$ channels. The first uncertainty is statistical and the second is systematic.

\begin{tabular}{|c|c|c|c|c|}
\hline low- $m_{j j}$ VR & $e e$ & $\mu \mu$ & & $e \mu$ \\
\hline$Z+$ jets & $30 \pm 2 \pm 13$ & $58 \pm 3$ & 7 & \pm 1 \\
\hline$t \bar{t}$ & $21 \pm 1$ & $30 \pm 1$ & 73 & \pm 19 \\
\hline$W t$ & $2.4 \pm 0.2 \pm 0.6$ & $2.9 \pm 0.3 \pm 0.7$ & 6.8 & $\pm 0.4 \pm 1.6$ \\
\hline diboson_QCD & $3.3 \pm 0.3 \pm 0.4$ & $5.2 \pm 0.3 \pm 0.5$ & 13.4 & $\pm 0.4 \pm 1.7$ \\
\hline diboson_EW & $0.0 \pm 0.0 \pm 0.1$ & $0.3 \pm 0.0 \pm 0.1$ & 0.6 & $\pm 0.0 \pm 0.1$ \\
\hline$Z \gamma$ & $4.3 \pm 0.4 \pm 1.4$ & $7.1 \pm 0.5 \pm 2.5$ & 0.1 & $\pm 0.1 \pm 0.1$ \\
\hline Higgs & $0.1 \pm 0.0 \pm 0.0$ & $0.3 \pm 0.0 \pm 0.1$ & 0.5 & $\pm 0.0 \pm 0.0$ \\
\hline$t t V$ & $0.0 \pm 0.0 \pm 0.0$ & $0.0 \pm 0.0 \pm 0.0$ & 0.1 & $\pm 0.0 \pm 0.0$ \\
\hline fake-lepton & $3.2 \pm 1.0 \pm 0.1$ & $0.0 \pm 0.0 \pm 0.0$ & 1.2 & $\pm 0.7 \pm 0.1$ \\
\hline Total background & $64 \quad \pm 3 \quad \pm 17$ & $103 \pm 3 \pm 29$ & 103 & $\pm 2 \pm 21$ \\
\hline Data & 51 & 95 & & 118 \\
\hline
\end{tabular}

from $10 \%$ to $70 \%$. The dominant sources come from uncertainties on JES, JER, $b$-tagging, and $E_{T}^{\text {miss }}$ modelling.

\section{Results}

The observed number of events in the signal region is compared to the expected background yield with both statistical and systematic uncertainties in Table 3 for all three channels. Signal yields for all resonance types with a mass of $300 \mathrm{GeV}$ are also shown.

Table 3. Signal and background yields [17] in the signal region for the $e e, \mu \mu$ and $e \mu$ channels. The first uncertainty is statistical and the second is systematic.

\begin{tabular}{|c|c|c|c|c|}
\hline & & $e e$ & $\mu \mu$ & $e \mu$ \\
\hline$Z+$ jets & 17.6 & $\pm 1.2 \pm 11.6$ & $36.6 \pm 2.3 \pm 19.0$ & $6.7 \pm 1.2 \pm 1.7$ \\
\hline$t \bar{t}$ & 12.1 & $\pm 0.6 \pm 3.2$ & $18.2 \pm 0.7 \pm 4.6$ & $46.9 \pm 1.2 \pm 12.1$ \\
\hline$W t$ & 1.2 & $\pm 0.2 \pm 0.3$ & $1.5 \pm 0.2 \pm 0.5$ & $3.1 \pm 0.3 \pm 0.8$ \\
\hline diboson_QCD & 3.1 & $\pm 0.3 \pm 0.5$ & $4.2 \pm 0.3 \pm 0.7$ & $10.2 \pm 0.3 \pm 1.6$ \\
\hline diboson_EW & 1.2 & $\pm 0.1 \pm 0.1$ & $1.7 \pm 0.1 \pm 0.2$ & $3.6 \pm 0.1 \pm 0.4$ \\
\hline$Z \gamma$ & 2.1 & $\pm 0.3 \pm 0.6$ & $3.8 \pm 0.3 \pm 0.7$ & $0.1 \pm 0.0 \pm 0.1$ \\
\hline Higgs & 0.3 & $\pm 0.0 \pm 0.1$ & $0.4 \pm 0.0 \pm 0.1$ & $0.8 \pm 0.0 \pm 0.1$ \\
\hline$t t V$ & 0.0 & $\pm 0.0 \pm 0.0$ & $0.0 \pm 0.0 \pm 0.0$ & $0.1 \pm 0.0 \pm 0.0$ \\
\hline fake-lepton & 0.6 & $\pm 0.6 \pm 0.1$ & $0.0 \pm 0.0 \pm 0.0$ & $1.3 \pm 0.7 \pm 0.1$ \\
\hline$\sigma(m=300 \mathrm{GeV})$ & 5.1 & $\pm 0.3 \pm 0.6$ & $7.5 \pm 0.3 \pm 0.9$ & $14.4 \pm 0.4 \pm 1.9$ \\
\hline$\phi(m=300 \mathrm{GeV})$ & 0.3 & $\pm 0.1 \pm 0.2$ & $1.0 \pm 0.1 \pm 0.4$ & $1.6 \pm 0.2 \pm 0.4$ \\
\hline$\rho(m=300 \mathrm{GeV})$ & 8.0 & $\pm 0.4 \pm 1.6$ & $11.7 \pm 0.4 \pm 1.4$ & $24.1 \pm 0.6 \pm 3.1$ \\
\hline$f(m=300 \mathrm{GeV})$ & 15.6 & $\pm 0.6 \pm 1.9$ & $22.6 \pm 0.8 \pm 1.9$ & $50.4 \pm 1.2 \pm 3.8$ \\
\hline$t(m=300 \mathrm{GeV})$ & 3.3 & $\pm 0.2 \pm 0.4$ & $4.7 \pm 0.2 \pm 0.6$ & $6.9 \pm 0.3 \pm 1.1$ \\
\hline Total background & 38.2 & $\pm 1.6 \pm 13.9$ & $66.4 \pm 2.5 \pm 21.6$ & $72.6 \pm 1.9 \pm 14.8$ \\
\hline Data & & 40 & 74 & 86 \\
\hline
\end{tabular}


Figure 2 shows the observed transverse mass distribution of the $W W$ system, $M_{\mathrm{T}}^{W W}$, compared to the SM prediction in the signal region for all three channels. The definition of $M_{\mathrm{T}}^{W W}$ is as follows:

$$
\left(M_{\mathrm{T}}^{W W}\right)^{2}=\left(P_{\ell_{1}}+P_{\ell_{2}}+P^{\mathrm{miss}}\right)\left(P_{\ell_{1}}+P_{\ell_{2}}+P^{\mathrm{miss}}\right),
$$

where $P^{\text {miss }}$ is the four vector defined by $\left(E_{T}^{\text {miss }}, E_{x}^{\text {miss }}, E_{y}^{\text {miss }}, 0\right)$. This definition is not a transverse mass variable in the classical meaning as it includes the longitudinal momenta of the two visible leptons. It is the sum of four-momenta of the two charged leptons and missing transverse momentum.
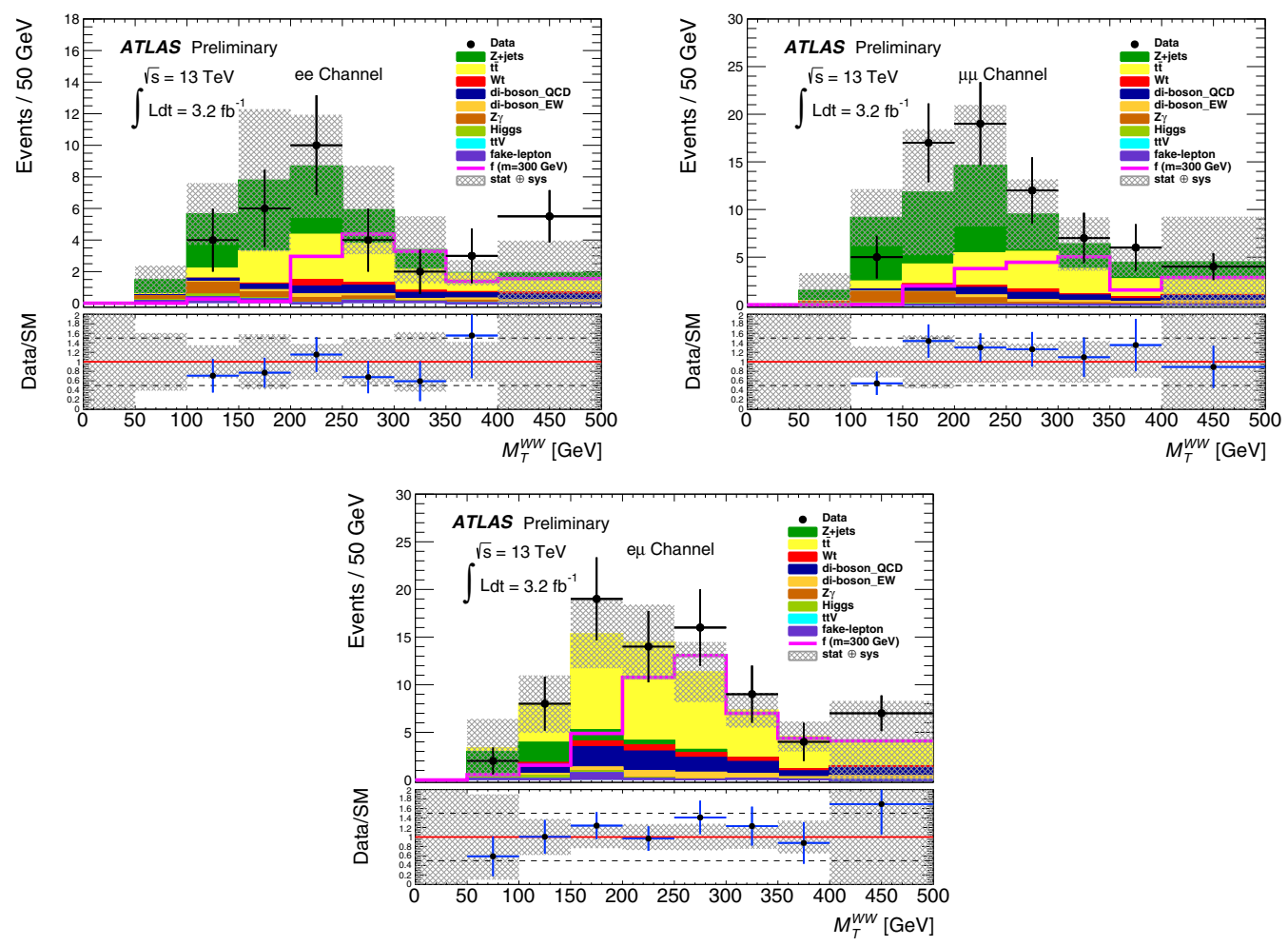

Figure 2. Observed and predicted $M_{\mathrm{T}}^{W W}$ distribution [17] in the $e e, \mu \mu$, and $e \mu$ channels in the signal region. The distribution for the $f$ resonance with $m=300 \mathrm{GeV}$ is also shown, and the hatched area indicates the overall uncertainty on the SM predictions. The lower plot for each decay mode shows the ratio of the data to the SM backgrounds and the hatched area represents the overall uncertainty on the ratio. The last bin includes overflow.

No excess is observed in data and $95 \%$ confidence level (C.L.) upper limits are therefore derived on the production cross section times branching ratio for these five new resonances $(\sigma q q q R q q \rightarrow$ $\left.\ell^{+} v \ell^{-} \bar{v} q q\right)$ with $\left.\ell=e, \mu\right)$. Due to limited simulated signal sample size at high mass, a counting experiment is performed using the observed data, SM background and signal events in the signal region. A combined likelihood function is constructed from the likelihood functions of each individual channel, given by Poisson distributions describing an event counting experiment for each channel, and global constraints for the set of nuisance parameters, which parametrize effects from systematic uncertainties. This treatment allows full accounting of the correlation of systematic uncertainties across various contributions (signal and backgrounds) and across channels. The upper limits on the 
production cross section times branching ratio are calculated from the profile-likelihood-ratio test statistic [40].

Figure 3 shows the expected and observed limits at 95\% C.L. as a function of the resonance mass, together with the \pm 1 and \pm 2 standard deviation uncertainty bands. The observed limits are found to be in the range of $380-220 \mathrm{fb}$ for the $\sigma$ particle, $460-240 \mathrm{fb}$ for the $\phi$ particle, $330-270 \mathrm{fb}$ for the $\rho$ particle, $340-260 \mathrm{fb}$ for the $f$ particle, and $310-260 \mathrm{fb}$ for the $t$ particle. These limits are for the resonance mass in the range of $200-500 \mathrm{GeV}$. With a coupling of 2.5 to longitudinal vector bosons, $\rho(f)$ particles with a mass below $230(300) \mathrm{GeV}$ are excluded at 95\% C.L.
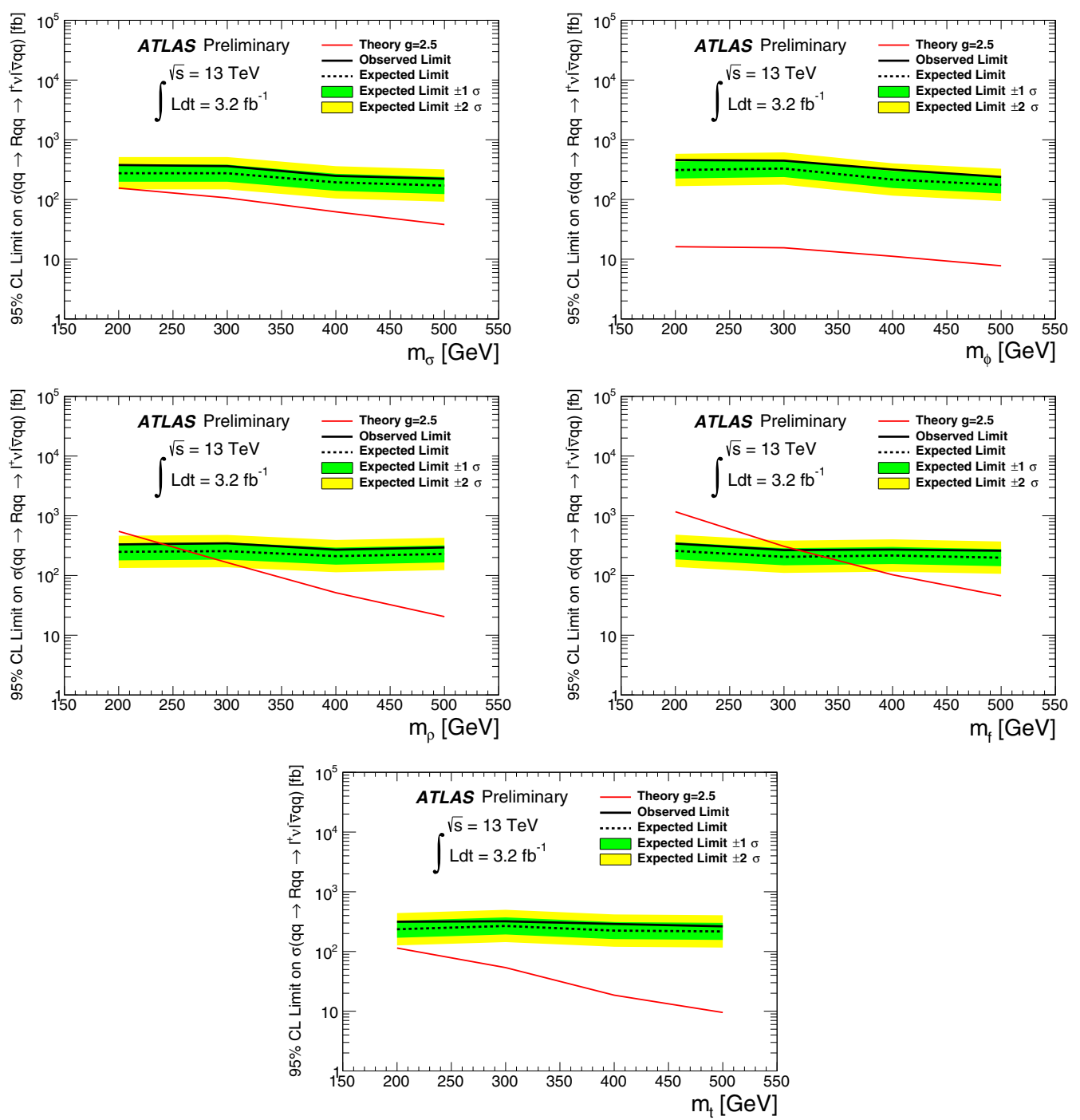

Figure 3. Expected and observed 95\% C.L. upper limits [17] on the $\sigma, \phi, \rho, f$ and $t$ production cross section times branching ratio, $\sigma\left(q q \rightarrow R q q \rightarrow \ell^{+} v \ell^{-} \bar{v} q q\right)$ with $\ell=e, \mu$, as a function of its mass. The expected limits are plotted with the \pm 1 and \pm 2 standard deviation uncertainty bands. 


\section{Conclusion}

Studies of vector boson fusion above the Higgs boson mass are important for a better understanding of the electroweak symmetry breaking mechanism. A search was performed for heavy neutral resonances produced through vector boson fusion $q q \rightarrow R q q \rightarrow \ell^{+} v \ell^{-} \bar{v} q q(\ell=e, \mu)$ using $3.2 \mathrm{fb}^{-1}$ of data at $\sqrt{s}=$ $13 \mathrm{TeV}$ recorded by the ATLAS detector at the LHC. No excess above the SM background expectation is observed. First sets of limits are obtained on the production cross section times branching ratio of five types of new resonances with different spins and isospins using $K$-matrix unitarization of the vector boson scattering process. The observed cross section limits are found to be in the range of $460-220 \mathrm{fb}$ for resonance masses from 200 to $500 \mathrm{GeV}$. For an assumed coupling of 2.5 to longitudinal vector bosons, $\rho$ and $f$ particles with a mass below $230 \mathrm{GeV}$ and $300 \mathrm{GeV}$ respectively are excluded at $95 \%$ C.L.

\section{References}

[1] M. J. Dugan, H. Georgi and D. B. Kaplan, Nucl. Phys. B254, 299 (1985)

[2] K. Agashe, R. Contino and A. Pomarol, Nucl. Phys. B719, 165 (2005)

[3] J. F. Gunion et al., Phys. Rev. D40, 1546 (1989)

[4] M. S. Chanowitz and M. Golden, Phys. Lett. B165, 105 (1985)

[5] J. F. Gunion, R. Vega and J. Wudka, Phys. Rev. D42, 1673 (1990)

[6] L. Randall and R. Sundrum, Phys. Rev. Lett. 83, 3370 (1999)

[7] H. Davoudiasl, J. L. Hewett and T. G. Rizzo, Phys. Rev. D63, 075004 (2001)

[8] D. Espriu and B. Yencho, Phys. Rev. D87, 055017 (2013)

[9] D. Espriu and F. Mescia, Phys. Rev. D90, 015035 (2014)

[10] W. Kilian et al., Phys. Rev. D91, 096007 (2015)

[11] A. Dobado, F.-K. Guo and F. J. Llanes-Estrada, Commun. Theor. Phys. 64, 701 (2015)

[12] T. Appelquist and G.-H. Wu, Phys. Rev. D48, 3235 (1993)

[13] W. Kilian, Springer Tracts Mod. Phys. 198, 1 (2003)

[14] A. Alboteanu, W. Kilian and J. Reuter, J. High Energy Phys. 11, 010 (2008)

[15] J. Schumacher, PhD thesis: TU Dresden, CERN-THESIS-2010-140, 2010, https://inspirehep.net/record/886924/

[16] W. Kilian et al., Phys. Rev. D93, 036004 (2016)

[17] ATLAS Collaboration, ATLAS-CONF-2016-053, 2016, http://cds.cern.ch/record/2206135

[18] ATLAS Collaboration, J. Instrum. 3, S08003 (2008)

[19] W. Kilian, T. Ohl and J. Reuter, Eur. Phys. J. C71, 1742 (2011)

[20] J. Reuter et al., J. Phys. Conf. Ser. 608, 012063 (2015)

[21] S. Alioli et al., J. High Energy Phys. 1006, 043 (2010)

[22] J. Alwall et al., J. High Energy Phys. 07, 079 (2014)

[23] T. Gleisberg et al., J. High Energy Phys. 02, 007 (2009)

[24] S. Catani et al., Phys. Rev. Lett. 103, 082001 (2009)

[25] R. Gavin et al., Comput. Phys. Commun. 182, 2388 (2011)

[26] K. Melnikov and F. Petriello, Phys. Rev. D74, 114017 (2006)

[27] M. Czakon and A. Mitov, Comput. Phys. Commun. 185, 2930 (2014)

[28] S. Agostinelli et al., Nucl. Instrum. Meth. A506, 250 (2003) 
[29] ATLAS Collaboration, Eur. Phys. J. C70, 823 (2010)

[30] ATLAS Collaboration, ATL-PHYS-PUB-2015-026, 2015, https://cds.cern.ch/record/2037717

[31] ATLAS Collaboration, ATLAS-CONF-2016-024, 2016, http://cds.cern.ch/record/2157687

[32] ATLAS Collaboration, Eur. Phys. J. C76, 292 (2016)

[33] M. Cacciari, G. P. Salam and G. Soyez, J. High Energy Phys. 04, 063 (2008)

[34] ATLAS Collaboration, Eur. Phys. J. C75, 17 (2015)

[35] ATLAS Collaboration, ATLAS-CONF-2014-018, 2014, http://cds.cern.ch/record/1700870

[36] ATLAS Collaboration, ATLAS-PHYS-PUB-2015-022, 2015, http://cds.cern.ch/record/2037697

[37] ATLAS Collaboration, ATL-PHYS-PUB-2015-027, 2015, https://cds.cern.ch/record/2037904

[38] ATLAS Collaboration, Phys. Rev. D92, 012006 (2015)

[39] ATLAS Collaboration, Phys. Rev. D90, 052005 (2014)

[40] ATLAS and CMS Collaborations, ATL-PHYS-PUB-2011-011, 2011, https://cds.cern.ch/record/1375842 